\title{
Temperature and Humidity Monitoring System Based On Adaptive Con- trol Strategy State Machine Model
}

\author{
Zhou-yafeng*
}

\author{
Electronics Information Engineering, Nanjing College of Information Technology, Nanjing, 210023, China
}

\begin{abstract}
By studying the current temperature and humidity monitoring system status, a kind of monitoring system of adaptive control strategy state machine model is proposed. The system uses network to connect server and sub control device and sub control device uses RS485 to connect temperature and humidity information collection and control terminal. By logging in management system, users can choose a suitable control strategy to control temperature and humidity basing on different demand and situation. Sub control device uses ARM9 of S3C2410 processor as the master chip, while temperature and humidity information collection and control terminal use AT89S51 as the master chip. To ensure the efficiency and scalability of network communication, to make it easy to build a distributed monitoring system, adaptive control strategy state machine model is used to build data path between server and sub control device. In strategy state machine model control, automatic control strategy can be written to an XML file and sent to the sub control device by the ZeroMQ to guarantee that even the server was broken down or it was no sever situation, the system can still work normally.
\end{abstract}

Keywords: Temperature and humidity monitoring, adaptive control strategy, ARM, ZeroMQ.

\section{INTRODUCTION}

The application field of temperature and humidity monitoring and supervision has a wide range, which is mainly in industry and agriculture, pharmaceuticals industry and electric power industry, etc. For instance, in the process of grain stock, to ensure the quality of grain, accurately monitoring the temperature and humidity of grain storehouse is demanded; in an unattended transformer substation, temperature and humidity are the main control parameters to control the appearance of condensation phenomenon. At present, on the basis of application demand of different businesses, many kinds of temperature and humidity monitoring systems are in use, which are used by utilizing computer, sensor, microprocessor, and network techniques instead of simple manual operation. They can intelligently control controlling devices basing on respective situation, such as air conditioning, dehumidifier, air-exhaust fan, etc. to meet the aim of energy conservation, reliability and manpower conservation.

With the recent development, temperature and humidity monitoring system is now taking on a tendency of diversification. On the basis of whether it is net-connected or not, it can generally be divided into two categories: 1) networking temperature and humidity monitoring system; 2) nonnetworking temperature and humidity monitoring system. The implementation of the first category can reference document [1-3]. It adopts network to transmit temperature and humidity monitoring information, and it improved scalability and flexibility of the system and at the same time the monitoring distance is developed, which is the present main stream monitoring system mode. The second system can be classified by whether it is connected with computer. The implementation of computer-connected system can reference document [4]. The key feature of the system is that the monitoring range is small, and it can directly uses RS485 or RS232 to feedback transmission data to computers. Noncomputer system, however, directly uses microprocessor to control and the implementation of it can reference [5], the feature of which is that it is true of small site and situations of low-need controlling.

The systems mentioned above have one common feature in the process of controlling--that is--the simplification of the controlling strategy. The difficulty of flexibly controlling on the basis of specific operation site and environment results in the limitation of system application. In this paper, the implemented adaptive control strategy system can control diverse devises basing on temperature and humidity, setting diverse temperature and humidity controlling demand in different rooms, assort-use intelligent control and manual control, etc., which widened application range and improved controlling flexibility and accuracy of the system.

\section{SYSTEM OVERALL STRATEGY}

The system is made up of for parts: server, client side, sub control device and temperature \& humidity information collection and control terminal (below uses abbreviation terminal).

The system overall diagram as showed in Fig. (1): 


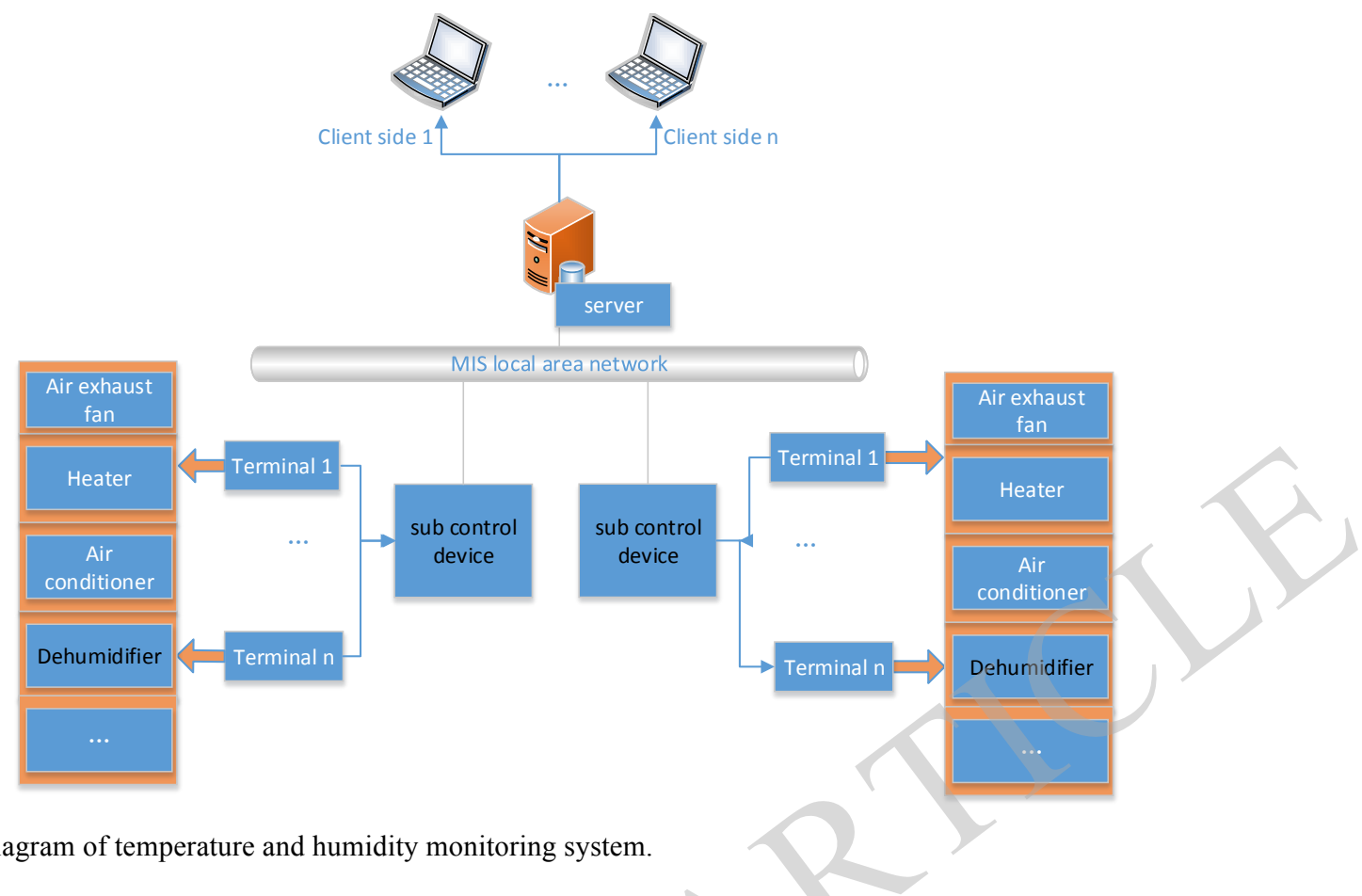

Fig. (1). The overall diagram of temperature and humidity monitoring system.

Server has built a management system basing on $\mathrm{B} / \mathrm{S}$ frame to make come true the interaction between server and client-side. At the same time, it has built a data transceiver program basing on $\mathrm{C} / \mathrm{S}$ frame to take charge the information transmission between sever and sub control devise.

Client logs in management system built by sever through client-side, finish initial work such as setting the initial information and parameter of the system, etc., type in the needed controlling strategy parameters to build basic controlling strategy mode, and then send relevant controlling parameters or files to sub control device by selecting controlling strategy.

Sub control device plays the rule like gateway, taking charge of collecting temperature and humidity monitoring data and management terminal, meanwhile, it receives and transmits information such as control command sent by server.

Terminal takes charge of transmitting data collected by temperature and humidity monitoring data sensor into numbers and send it to sub control device through RS485, meanwhile, it transmits it into level signal or infrared signal to control controlling devices. This structure is widely used in the monitoring system [6].

\section{ADAPTIVE CONTROL STRATEGY}

Definition of State Machine: Full name is finite-state machine, FSM), is a finite-state and between these States and movements and the transfer of mathematical models of behavior. Using Mealy state machine, its next State, known as the output is determined by the input and current state [7,8]. The article use Mealy State Machine, its next State is determined by the input and output the current state. Based on State of the state machine described considered a one-way flow, that is, from the start State to the termination status changes from acyclic directed graph to describe the process. State machine consists of five-tuples $\left(\Sigma, S, s_{0}, \sigma, F\right)$, and their meanings are as follows:

$\Sigma$ : indicates enter a non-empty finite set, the corresponding system set of trigger conditions;

$S$ :Means status of non-empty finite set, corresponding to the current state of the system set and output status set ;

$s_{0}$ : indicates Initial state $\left(s_{0} \in S\right)$;

$\sigma$ : indicates representing a state transition function $(\sigma: S \times \Sigma \rightarrow S)$. That triggered the current state of the system set change output state under the conditions of collection;

$F$ : indicates a collection of final, reached the final again after transfer to other state $(F \subseteq S$ ).

Adaptive Control Strategy P: Adaptive control strategy refers to the temperature and humidity monitoring system in order to ensure the enforceability of, when facing multiple triggering events to execute for each device control rule set. This article describe the variable policy to $P \leq D, C, R$. D represents control field, $\mathrm{C}$ represents trigger conditions, $\mathrm{R}$ is Contains a rule set. Each rule in the rule set $r$ corresponds to a configurable action. Based on finite state machine model of a rule $\mathrm{r}$ is defined as $r=<i, o, s_{0}, l>$, Where $i$ represents the original state rules, $O$ represents an output state. The State in 


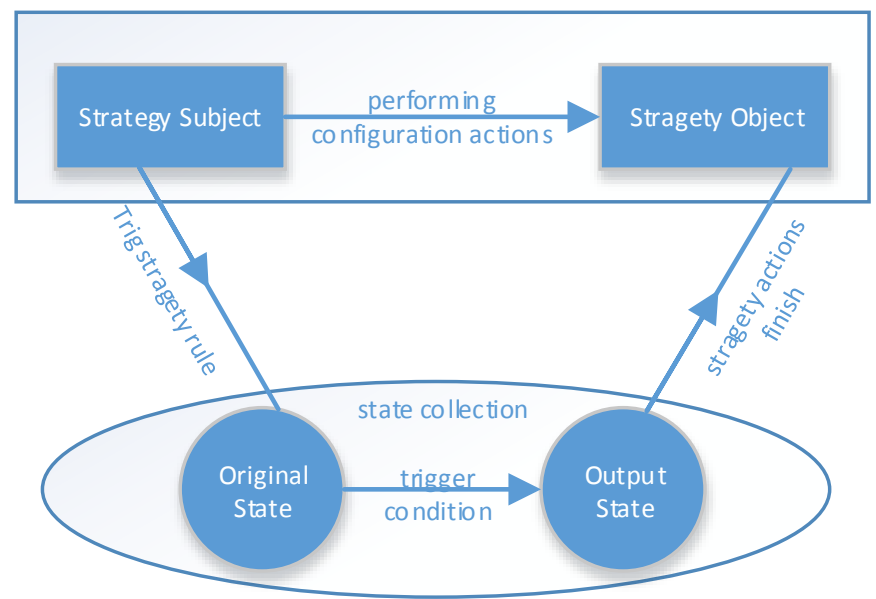

Fig. (2). Adaptive implementation of the strategy process.

the state machine can be described as $i(c) \rightarrow o$, of which $i, o \in S, c \in \sum$. For example, "Open air" for OpAir, the action of "cold", then the system into executing the rule "reach the required temperature opens the air conditioning refrigeration" (recorded as DoCold), then this policy rule can be represented as OpAir (Cold) $\rightarrow$ DoCold . $s_{0}$ indicates that the rule's initial state. $l$ indicates whether the output state of the rule as final. Because the output state of the rules with policy enforcement action to achieve the effect, it is no longer explicitly shows policy takes action. Each policy contains one or more rules, each one corresponding to a unique state in the state machine process, which contains a configuration that contains only complete the configuration of a state change. Because the adaptive control strategy is a set of rules, so long as the current state of the system and trigger conditions are met, a number of non-contradiction in the rules configuration actions can be performed simultaneously. Adaptive control strategy process implementation as shown in Fig. (2).

\section{SERVER PROGRAM DESIGNING}

Server B/S frame management program contains features as follow:

User's permission and limitation management module: It is used for managing user's implementation and operation privilege in the system, including super manager, general manager, and general user. Through classification the implementation and operation privilege of different users are set, so that the mistaken operation leading to accident is reduced.

Initial information typing-in module: It is user that is to type in the initial information the system operation needs, including system room information, sub control device information, terminal information, infrared control bank information, relevant parameter of controlling mode setting, device type and possible situation information typing-in, different controlling command pair information of different devices, etc.
Database module: It is mainly used for supporting users to check the current device operation situation, terminal collection of temperature and humidity live data and curves, historical data report, etc.

Strategy document generation module: It is used in the situation of sending system strategy document to sub control device. According to present system operation situation typed in by users, on the basis of the number of sub control device and relevant room, terminal information connected by sub control device and the present device strategy, the XML form strategy control document is made up, and finally it will be sent by program under $\mathrm{C} / \mathrm{S}$ mode to sub control device so that the its analysis can be operated.

Strategy bank module: This module is the key module of the system and all of the system temperature and humidity controlling strategy is relevant to it. The general classification status of the controlling strategy are described in Table $\mathbf{1 .}$

Table 1. System controlling strategy.

\begin{tabular}{|l|l|}
\hline \multicolumn{1}{|c|}{$\begin{array}{c}\text { The Name of Controlling } \\
\text { Strategy }\end{array}$} & \multicolumn{1}{c|}{ Function } \\
\hline \hline Manual controlling strategy & $\begin{array}{l}\text { Users control device operating condi- } \\
\text { tion through managing systematic } \\
\text { manual controlling }\end{array}$ \\
\hline $\begin{array}{l}\text { The controlling strategy } \\
\text { based on devices }\end{array}$ & $\begin{array}{l}\text { On the basis of the given system tem- } \\
\text { perature and humidity controlling } \\
\text { command, control the system taking the } \\
\text { device form as a unit. }\end{array}$ \\
\hline $\begin{array}{l}\text { The controlling strategy } \\
\text { based on rooms }\end{array}$ & $\begin{array}{l}\text { It can set different temperature and } \\
\text { humidity controlling command of dif- } \\
\text { ferent rooms and manage all the con- } \\
\text { trolling devices of the room taking the } \\
\text { room as a unit. }\end{array}$ \\
\hline $\begin{array}{l}\text { The controlling strategy } \\
\text { based on server }\end{array}$ & $\begin{array}{l}\text { Server take control of the system, using } \\
\text { historical data information, adopting } \\
\text { more complicated controlling mode } \\
\text { based on neural network etc. }\end{array}$ \\
\hline
\end{tabular}




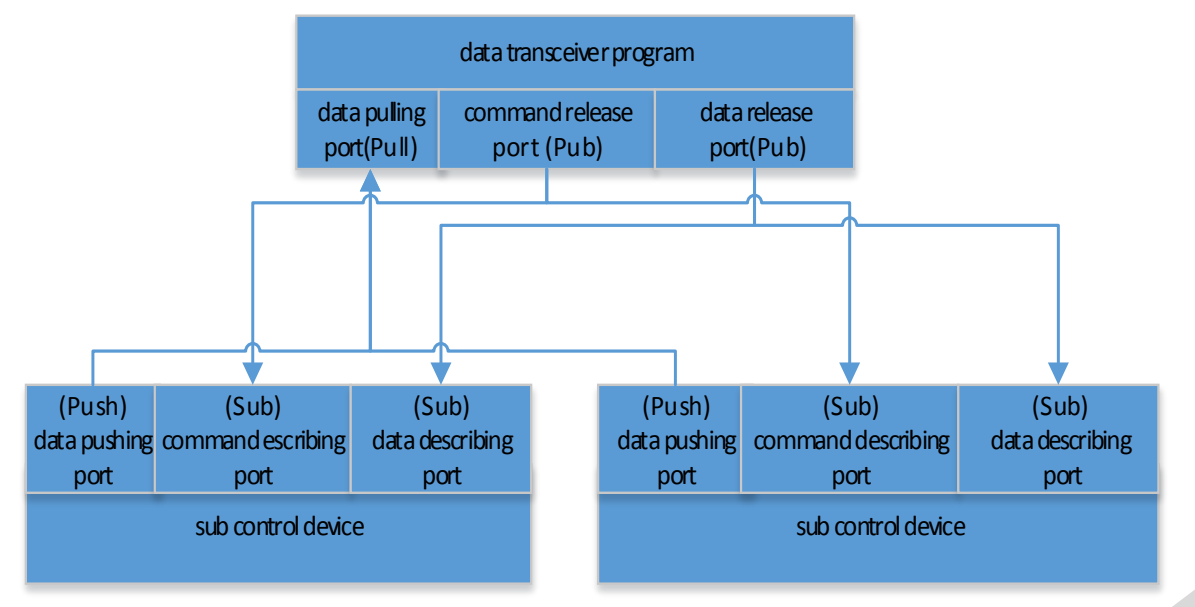

Fig. (3). ZeroMQ communication mode designing.

The controlling strategy based on devices and the controlling strategy based on rooms are the kind of controlling strategies that can create relevant controlling strategy document. They create strategy controlling document by strategy document creating module, and they send the strategy document to sub control device to take control of the controlling device through $\mathrm{C} / \mathrm{S}$ data transceiver program. It is just because this that under the two kinds of strategy module the system can operate normally when breaking away from server.

At the same time, to further improve the flexibility of the system controlling, the system management concerning the above controlling strategy is allowed to some degree. For instance, when room A takes control by using controlling strategy based on devices, one or more devices in the room can be set as excluded items and give them to manual controlling strategy for management; or if in the system the room A takes control by using controlling strategy based on devices or controlling strategy based on rooms, meanwhile the room B, which has a high command of controlling accuracy, the system takes control by adopting the controlling strategy based on server.

By flexibly combining different controlling strategies, the system controlling particle size will be smaller and the system can be more flexible. House temperature control strategy can reference to [9].

Server C/S frame data transceiver program adopts crossplatform Qt technology to develop. Its main function of the accomplishment is sending device information under the frame of $\mathrm{B} / \mathrm{S}$ to the sub control device, meanwhile, typing the terminal information collected by sub control device in database. The communication bank that is adopted to communicate with sub control device is ZeroMQ. ZeroMQ is a kind of open source, cross-platform, high performance, concise and flexible network information bank or a kind of middleware. It shields basement abnormal such as broken connection, data packet loss, etc. in the process of communication and it make users to concentrate their experiences more on the accomplishment of business logic. The four kinds of supportive communication modes are: request response mode, release/subscribe mode, pipeline mode and signal mode. The more information can be referred to the official website $[10,11]$. The distributed system accomplishment designed by ZeroMQ can be referred to document [12].

The operational mode during the process of data transceiver program is showed as Fig. (3):

In the system, three communication channels are built using ZeroMQ, the first is temperature and humidity information channel of Pull-Push mode; the second is command Pub-Sub mode channel that is used for server's sending controlling command to sub control

Device; data Pub-Sub mode channel is used for sending strategy document to sub control device.

\section{SUB CONTROL DEVICE DESIGN}

The system adopts $\mathrm{S} 3 \mathrm{C} 2410$ chip whose key is ARM920T as the main control chip of the sub control device. The chip is lower in price when compared with S3C2440. What he system need concerning outskirts devices contain network card and serial port, among which the serial port manage the system resources through clipped submersible Linux system on sub control device. According to program function, the sub control device program can be divided into the following modules:

XML strategy document analysis module: it is used for analyze XML strategy controlling document created by server.

Clocking roll polling module: according to parameters and terminal information set by XML strategy document, it roll-poll timing terminal that is connected with sub control device, and then collect temperature and humidity information.

Serial port communication module: it analyzes command document sent by terminal and then send it to the terminal. To speed up the rate of analyzing command document, it adopts limited state machine to speed up the process of 


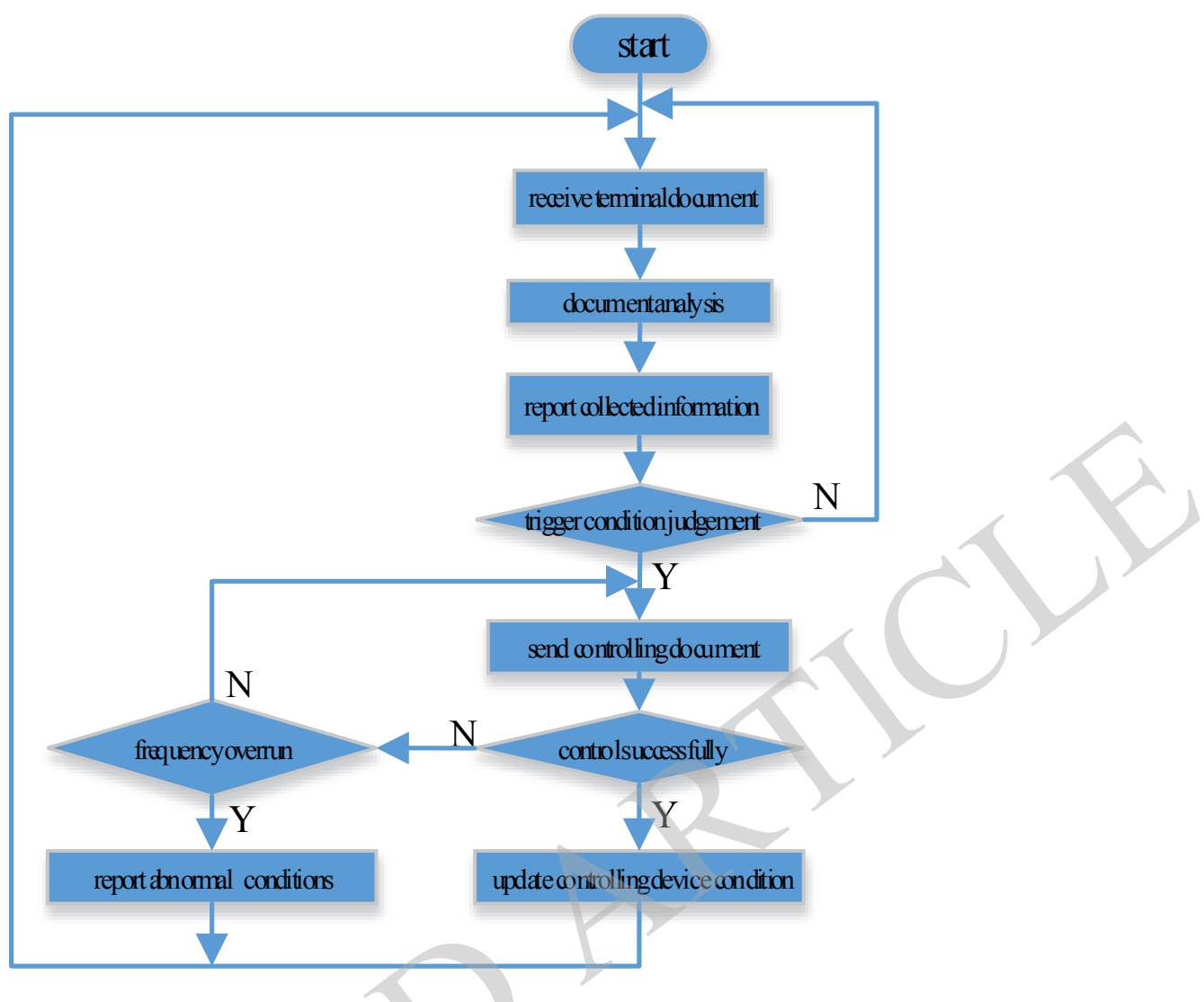

Fig. (4). The control procedure with strategy document.

analysis. Meanwhile, because the limited state machine relies on status value, to avoid mistakes in analysis, simple filter bag function and the latter verification function is added before and after analysis to reduce communication analysis mistakes.

ZeroMQ communication module: To accomplish the three data channels mentioned above, define data structure during communication procedure, collect and package data and send them to server, receive command and data from server.

Mode switch module: according to command and data information sent by server, it updates system controlling strategy.

To make sub control device has higher efficiency during data processing and operation, Epoll mode in Linux is used for designing during the process of data communication, which increases the data handling capacity during the process of system communication and improved the response speed. Meanwhile, the subsequent content in the system, such as regular roll poll, operating control and so on are proceeded and subsequently-operated in the form of multithread. In the system, except the main tread, 3 working treads are defined to proceeding system management: 1) initial roll poll tread: it is used for terminal that is connected by roll poll sub control device and check terminal working condition and initial system; 2) timer incident roll polling: according to parameters set by timing roll polling mode in strategy document, regularly send checking document to terminal so that data information collected by terminal sensor can be again collected. 3) operation roll polling: according to terminal document information received and trigger parameter defined in strategy document, take charge of the to-control items that is lined up in queue. And to check the control results, we need to check whether the control is successful according to the maximum operation number defined in strategy document.

The working procedure of sub control device under the condition of adaptive control strategy as showed in Fig. (4):

As for other control strategy, the operation system is relatively easier, it only need to broadcast control command sent by server to the overall tread, then the terminal will receive and analyze command document according to the address. Here the detailed introduction are eliminated.

\section{TERMINAL DESIGNING}

The terminal is designed by adopting extendable plug form, the terminal address is set by using dial switch, and it adopts RS485 to communicate with outside world. The main devices can be divided into 2 categories and they can choose 


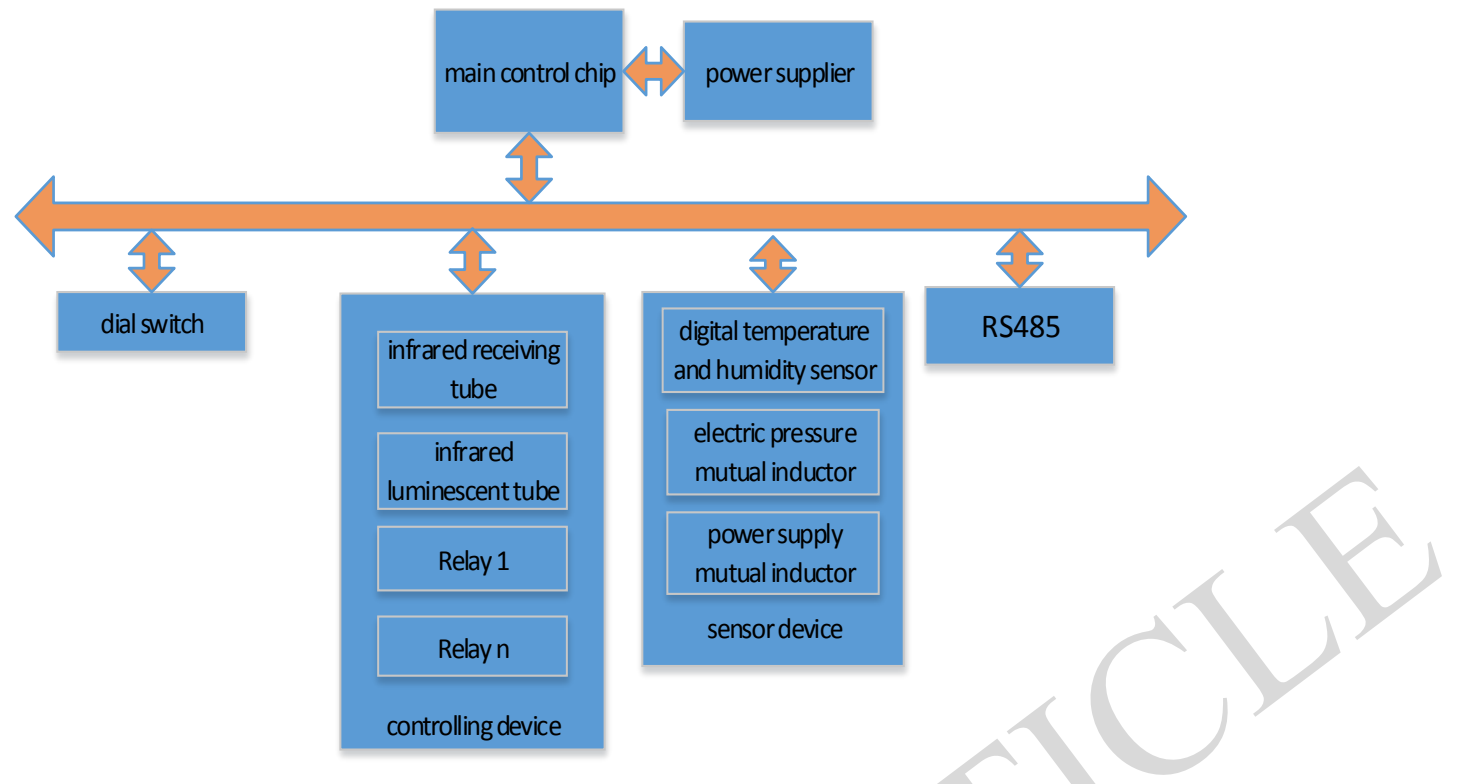

Fig. (5). Overall structure of terminal.

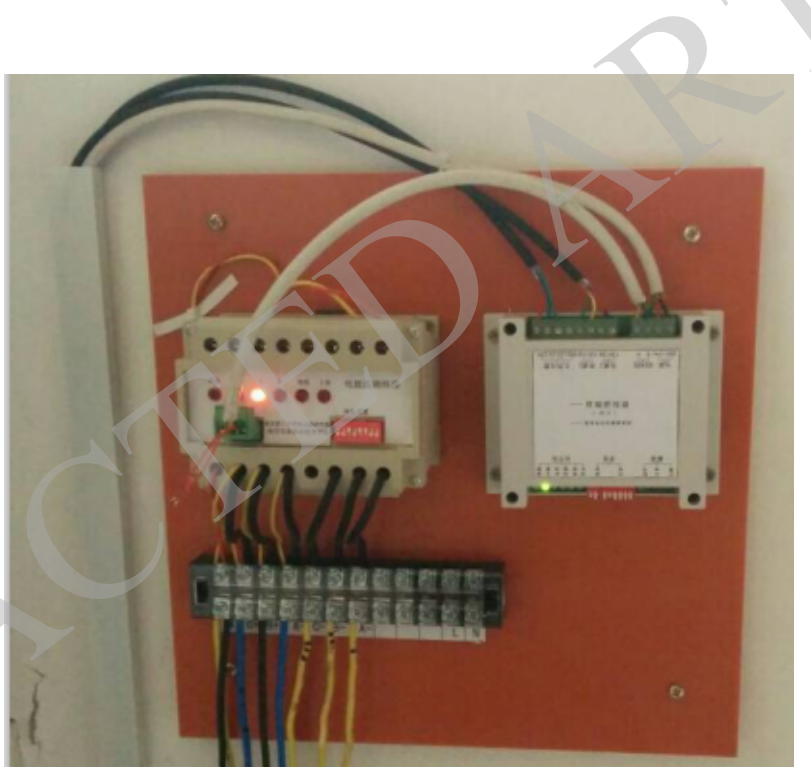

Fig. (6). Sub control device.

infrared form or through relay to control according to the outer device types. Except the basic temperature and humidity sensor, sensors can increase electric tension and current transformer to check the operating condition of control devices. The concrete structure is showed as Fig. (5).

\section{TESTING}

The system testing environment contains two rooms, it is respectively room $\mathrm{A}$ and room $\mathrm{B}$. The distance between room $\mathrm{A}$ and $\mathrm{B}$ is about 50 meters. In the room $\mathrm{A}$, there are 3 air conditioners, 2 dehumidifiers, and in room $\mathrm{B}$, there are 3 dehumidifiers and 4 air conditioners.

All the terminals RS485 are connected with sub control device in the form of parallel connection. The communication mode is active-standby mode. It is sub control device that begin the conversation and terminal responses, so that the highway conflict can be eliminated. In the on-site test, sub control device and server client side are both in the room C. To see according to testing condition, the accuracy rate of serial port communication is about $96 \%$, which basically meets the system requirement. The information maximum bandwidth between server and sub control device is $2 \mathrm{Mb} / \mathrm{s}$. Because of the condition limits, the maximum load of the system was not tested. During the function test, the room A and $\mathrm{B}$ are both set to different strategies and they both operate well and regularly.

Sub control device as shown in Fig. (6), the monitoring server as shown in Fig. (7), the monitoring center is currently used in one system for coal mining system, working after using the interface as shown in Fig. (8). 


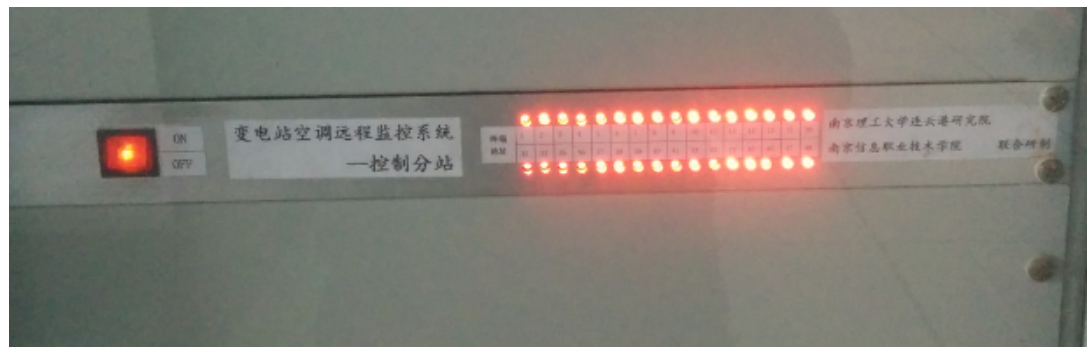

Fig. (7). Monitor server.

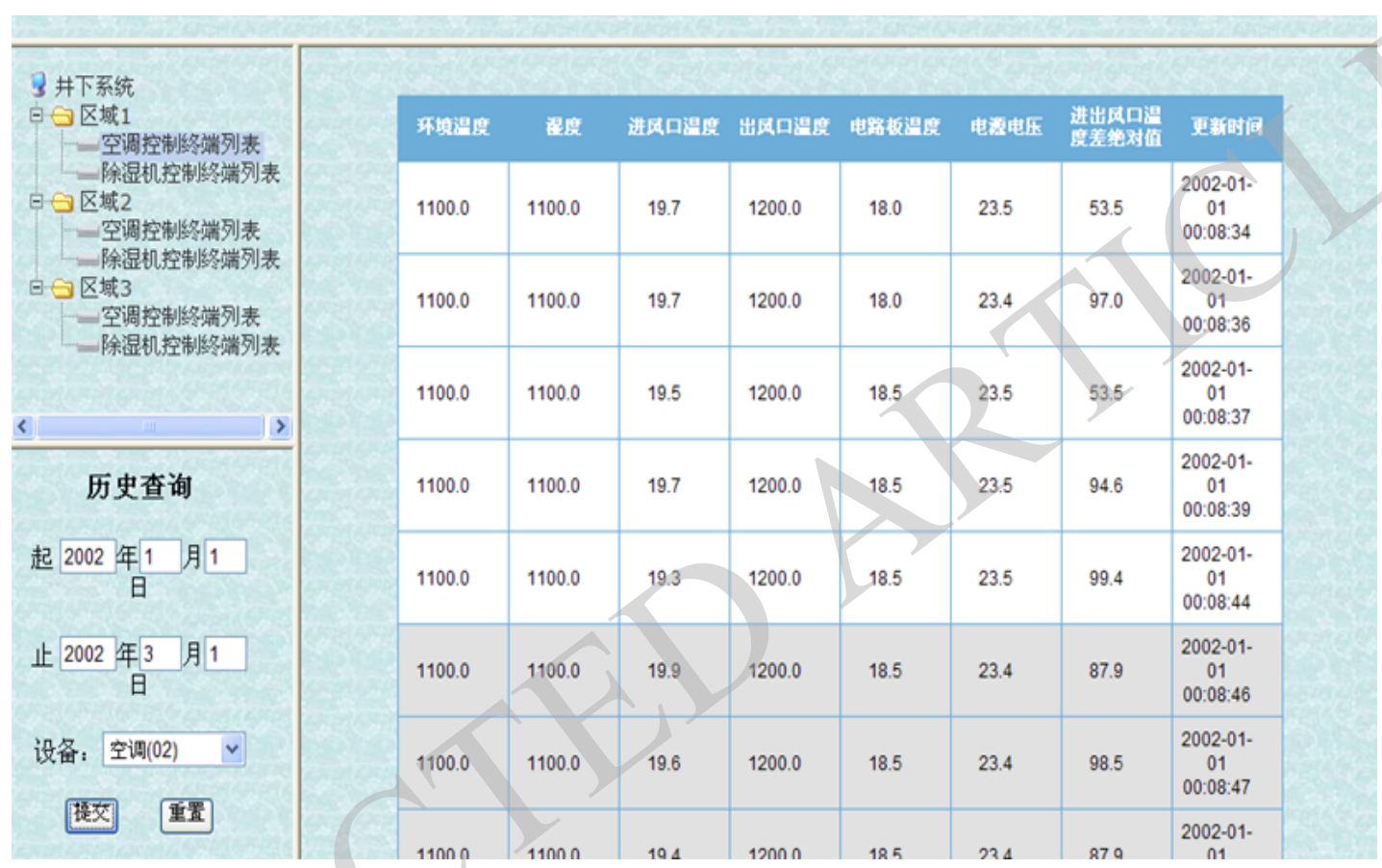

Fig. (8). Monitor website interface.

\section{CONCLUSION}

The above paper puts forward a kind of temperature and humidity monitoring of adaptive control strategy which contains four parts: server, client side, sub control device and terminal. In addition, through the device, under the circumstance of no server or damaged server, it can ensure the normal operation of the system. The feature of the system is that it base on adaptive control strategy state machine model to proceed information transmission of server and sub control device, it uses ZeroMQ to record controlling strategy, and it uses RS485 highway to connect sub control device and terminal; adaptive control strategy has the feature of adjustability and flexibility. It is tested that the system can provide a temperature and humidity monitoring that is more precise, flexible and reliable.

\section{CONFLICT OF INTEREST}

The author confirms that this article content has no conflict of interest.

\section{ACKNOWLEDGEMENTS}

Declared none.

\section{REFERENCE}

[1] L. Ming, F. Qiaoli, Y. Yehua., "The accomplishment of the distributed temperature and humidity monitoring system based on network," Information Technology, no. 11, pp. 103-105, 2013.

[2] G. Ke, Y. Wang, and S. Qi, "Network type temperature and humidity monitoring system," Electronic Designing Engineering, vol. 18 no. 4, pp. 41-43, 2010

[3] Q. Song, Z. Wei, and L. Zhang, "Temperature and humidity intelligent monitoring system based on ethernet distributed type," Controlling System, vol. 8, no. 17-19, 2007.

[4] Q. Liu, B. Jiao, and Y. Liu, "Warehouse temperature and humidity monitoring \& n rf905 wireless," Transmission System Designing, vol. 30, no. 5, pp. 101-103, 2011.

[5] Z. Liu, P. Guo, W. Zhang, and S. Ren, "Temperature and humidity monitoring system based on ARM," Instrument Technology and Sensor, no.12, pp. 108-110, 2009.

[6] A. Soetedjo, Y. Nakhoda, A. Lomi, F. Fahran, "Web-SCADA for monitoring and controlling hybrid Wind-PV power system," TELKOMNIKA (Telecommunication Computing Electronics and Control), vol. 12, no.2, pp. 305-314, 2014. 
[7] N. Chen, and J. Zhu, "A robust zero-watermarking algorithm for audio," EURASIP Journal on Advances in Signal Processing, vol. 2008, no. 103, pp. 103-110, 2008.

[8] L. Tan, B. Wu, Z. Liu, and M. Zhou, "An audio information on hiding algorithm with high capacity which based on chaotic and wavelet transform," Acta Electronic Sinica, vol. 38, no. 8, pp. 1812-1818, 2010.

[9] A. Alimuddin, K. B. Seminar, I.D.M. Subrata, N. Nomura, S. Sumiati, "Temperature control system in closed house for broilers based on ANFIS," TELKOMNIKA (Telecommunication Computing Electronics and Control), vol. 10, no. 1, pp. 75-82, 2012.

[10] M. Q. Zero, http://zeromq.org/,2014

[11] F. Pu, and J. Chen, "Distributed system based on ZeroMQ," Electronic Testing, no.7, pp. 24-29, 2012.

[12] S. Ye, and J. Yao, "Distributed monitoring system information communication frame designing based on ZeroMQ \& JSON," Modern Electronic Technology, vol. 37, no.2, pp. 105-109, 2014.

Received: September 16, 2014

(c) Zhou-yafeng; Licensee Bentham Open.

This is an open access article licensed under the terms of the Creative Commons Attribution Non-Commercial License (http://creativecommons.org/licenses/by$\mathrm{nc} / 3.0 /$ ) which permits unrestricted, non-commercial use, distribution and reproduction in any medium, provided the work is properly cited. 